sensitivity of both sodium and potassium channels to toxins. The most compelling of these investigations indicated that the sites noted in the figure ( $a$, right-hand side) interact closely with toxins in the extracellular medium and thus could be near the outside mouth of the pore.

Hartmann et al. $^{2}$ used these clues to exploit two different potassium channel clones that, when expressed in oocytes, exhibit different pore properties. The two clones differ in three functional characteristics: conductance of the open channel, and sensitivity to block by tetraethyl ammonium (TEA) ions from the outside and from the inside (the inside and outside TEA-binding sites were known to be distinct). When Hartmann et al. transplanted a region of $\mathrm{P}$ from the first channel type to the second - the chimaeric channel had nine amino-acid substitutions out of 21 residues - the channel properties followed the transplant without, amazing as it seems, there being other dramatic changes in channel function. Thus, these nine substitutions seemed to alter TEA blocking from both inside and outside, and to change single-channel conductance - just what one would expect if $P$ formed the pore.

If the region of $\mathrm{P}$ modified by Hartmann et al. really is the pore, then modest structural changes in this region should alter the selectivity to various ions. Potassium channels permit the passage of potassium ions and exclude sodium ions, but they also permit entry of two ions that have crystal radii larger than potassium ammonium $(0.14 \mathrm{~nm})$ and rubidium $(0.15$ $\mathrm{nm}$ ). Yool and Schwarz' found that two specific amino-acid substitutions within $\mathrm{P}$ modified the pore permeability to ammonium and rubidium without changing other properties of the channel. Neither of the substitutions made by Yool and Schwarz included residues altered by Hartmann et al., although they are in the same region.

These first two studies ${ }^{1.2}$ demonstrate that $\mathbf{P}$ is critically involved in ion permeation and selectivity, but they are less informative on the question of what amino-acid sequence actually spans the membrane: the changes made by Hartmann et al. changed something on the inside because they altered TEA sensitivity from the inside, but substituting nine amino acids might have produced significant structural modifications in neighbouring regions. Yellen $e t$ al ${ }^{3}$ have found, however, that the conservative threonine (T) to serine (S) mutation - a change that Yool and Schwarz found alters ionic selectivity - in the middle of $\mathrm{P}$ alters the blocking of the pore for inside, but not outside, TEA. So either P loops from the outside to the inside and back again, forming the pore, or the T-to-S mutation produces some structural changes that propagate to the inside, an extremely unlikely possibility for such a conservative substitution.

Few studies that employ site-directed mutagenesis have faced the issue of a mutation's sphere of influence - the distance over which structural perturbations propagate - and even crystallography, with its elucidation of frozen structures, cannot always provide a final solution. Nevertheless, the three studies described here convince me that we have the pore: P loops in and out, and forms the structure that permits ions to enter and leave the cell.

What are the next steps? Having identified the structure, we now need to work out how the amino acids are arranged in three dimensions, and how, or to what extent, this structure accounts for ionic selectivity. This is a hard problem which will not yield answers immediately, but we now have a firm place from which to start.

The new observations have at least two general implications. First, the striking thing about the swap made by Hartmann $e t$ $a l$. is how little other functions of the channel were modified. Their results indicate that one can switch in an entire function, the permeation of a channel, while keeping the bulk of the channel structure unchanged. The $\mathrm{P}$ region lies between a pair of introns, so I view the observations as supporting the notion that even quite fancy proteins can be constructed of modules. Second, it was predicted earlier, purely from an examination of the primary structure of channels ${ }^{7}$, that $P$ forms the pore. Unlikely as this might seem, the results summarized here give strong support to the idea that one can deduce unusual aspects of three-dimensional structure from the amino-acid sequence of a membrane protein. Of course, one does not know the limits of validity for such structural predictions - a question which needs intensive study but this victory by a predictor of structure may worry those who have previously dismissed such efforts out of hand.

When all is said and done, however, what we really need is high-resolution structures of channels. Those of us in the channel business feel that we are doing our part, and we wonder when the structural biologists are going to do theirs.

Charles F. Stevens is in the Molecular Neurobiology Laboratory, The Salk Institute, 10010 North Torrey Pines Road, La Jolla, California 92037, USA.

1. Yool, A. J. \& Schwarz, T. L. Nature 349, 700-704 (1991)

2. Hartmann, H. A. Drewe et al. Science 251, 942 (1991).

3. Yellen, G., Jurman, M., Abramson, T. \& Mackinnon, R. Science 251, 939-942 (1991)

4. Mackinnon, R \& Miller, C. Science 245, 1382-1385 (1989).

5. Noda, M. Suzuki, H., Numa, S. \& Stuhmer, W. FEBS Lett. 259, 213-216 (1989)

. Mackinnon, R., Heginbotham, L. \& Abramson, T. Neuron 5. $767-771$ (1990)

Guy, H. R. \& Seetharmulu, P. Proc. natn. Acad. Sci. U.S.A. $830,508-511$ (1986).

\section{Internal skin-care}

SOME chemicals, like the active principle of garlic, many compounds of tellurium, and dimethyl sulphoxide, can cross the skin barrier and enter the body by simple contact. Once inside, they can also come out again by the same route. Garlic fanciers and tellurium chemists tend to smell of their interests, as the odorous substances emerge relentlessly through the skin.

Daedalus is now trying to apply this principle. His first goal is an internal perfume'. Taken in pill form, it would break down in the body to a substance which sweated steadily out through the skin. Unlike conventional perfumes, it could not be washed off, but would deliver its odorous message evenly and uniformly for hours or even days at a time. Daedalus points out that many perfumes are derivatives or imitations of the subtle sex attractants of animals, which are of course glandular secretions designed to escape from the creature in just this way. So the principle seems fairly sound.

His second goal is an internal insect repellant. Some trees already have one: they protect themselves from insect attack by exuding hormone-like substances which disrupt the life-cycle of the pests. Many animals are tortured so mercilessly in their natural state by flies, mosquitoes and other insects that they too should have evolved a chemical defence. DREADCO biochemists are visiting the local zoo to take blood or sweat samples from giraffes, antelopes, wildebeeste and so on. They plan to chromatograph their specimens into components, and screen them for activity as skin-passing insect repellants or even insecticides. From the results, they hope to design a human version. In pill form, it will protect its swallower against the hungriest mosquito or most enraged wasp.

But DREADCO's main ambitions in this field depend on the decay of the ozone layer. The rising ultraviolet content of sunlight is spreading skin cancer among sunloving white-skinned people. An internal sun-blocking pill should be a real winner. Again, nature may have got there first. Chameleons, frogs and many other creatures react to the colours in their environment. Does their camouflage skill extend into the ultraviolet? If so, their skin chemistry might be adapted to human use. Daedalus imagines some photosensitive chemical which circulates harmlessly inside the body, but is vulnerable to ultraviolet light when it traverses the skin capillaries. Such radiation photolyses it and binds it to the skin at that point as a substance which itself absorbs ultraviolet strongly. It will thus migrate to, built up in, and protect the exposed regions of the skin. A little extra biochemical cunning might even arrange for it to confer a becoming $\tan$.

David Jones 